Archives de sciences sociales des religions

115 | juillet-septembre 2001

Islam et politique dans le monde (ex-)communiste

\title{
Islam et politique dans le monde (ex-)communiste
}

\section{Marc Gaborieau et Alexandre Popovic}

\section{CpenEdition}

\section{Journals}

Édition électronique

URL : http://journals.openedition.org/assr/17593

DOI : $10.4000 /$ assr. 17593

ISSN : $1777-5825$

Éditeur

Éditions de l'EHESS

Édition imprimée

Date de publication : 1 octobre 2001

Pagination : $5-13$

ISBN : 2-222-96707-4

ISSN : 0335-5985

Référence électronique

Marc Gaborieau et Alexandre Popovic, «Islam et politique dans le monde (ex-)communiste », Archives de sciences sociales des religions [En ligne], 115 | juillet-septembre 2001, mis en ligne le 19 août 2009, consulté le 20 avril 2019. URL : http://journals.openedition.org/assr/17593 ; DOI : 10.4000/assr.17593

Ce document a été généré automatiquement le 20 avril 2019

(C) Archives de sciences sociales des religions 


\title{
Islam et politique dans le monde (ex-)communiste
}

\author{
Marc Gaborieau et Alexandre Popovic
}

1 Ce numéro spécial ${ }^{1}$ compare les évolutions récentes des principales communautés musulmanes qui, des Balkans à la Chine en passant par l'Asie centrale, ont été, ou sont encore, sous des régimes communistes. Elles ont subi jusque dans un passé récent, ou subissent encore, un contrôle étatique extrêmement strict pouvant aller jusqu'à des tentatives de destruction des institutions religieuses comme ce fut le cas en URSS avant la Seconde Guerre mondiale, en Chine pendant la révolution culturelle, et en Albanie plus récemment encore. La libéralisation relative des régimes communistes dans les années 1980, y compris en Chine, puis la dislocation de l'URSS et l'effondrement des régimes communistes de Yougoslavie, de Bulgarie et d'Albanie, donnèrent aux communautés musulmanes de ces pays la possibilité de réaffirmer leur identité religieuse et de se réorganiser, pacifiquement ou dans la violence.

2 En mettant sur pied ce numéro les éditeurs poursuivent un double but : faire le point au cas par cas de la situation de l'islam dans chacune des régions considérées; puis voir si, au-delà de la disparité des situations, il n'est pas possible de dégager des tendances communes.

3 L'inventaire n'a pu malheureusement être exhaustif. Les exemples traités ici fournissent cependant une illustration des principaux cas de figure. La présentation a été ordonnée de façon à la fois géographique et historique. Elle commence par l'Asie orientale et centrale où les musulmans durent composer depuis des siècles avec l'expansion de deux grands empires rivaux, la Chine et la Russie : la première est encore communiste ; la seconde l'a été jusqu'en 1991, date où l'écroulement de l'URSS a redonné aux républiques musulmanes d'Asie centrale leur autonomie politique. L'Afghanistan, durement marqué par une brève occupation soviétique à partir de 1979, est traditionnellement lié à l'Asie centrale et traité ici avec elle. L'attention se déplace ensuite sur les minorités musulmanes qui entrèrent pour certaines dans la mouvance russe bien avant l'Asie centrale, et qui restent toutes englobées dans l'actuelle Russie au Tatarstan (dans la haute Volga) et dans le Caucase du Nord. L'on passe ensuite à l'Azerbaïdjan dans le Caucase du 
Sud qui est devenu indépendant après l'écroulement de l'URSS. L'inventaire se termine par des pays européens qui tombèrent sous domination communiste après la Seconde Guerre mondiale : la Bulgarie, l'Albanie et la Yougoslavie.

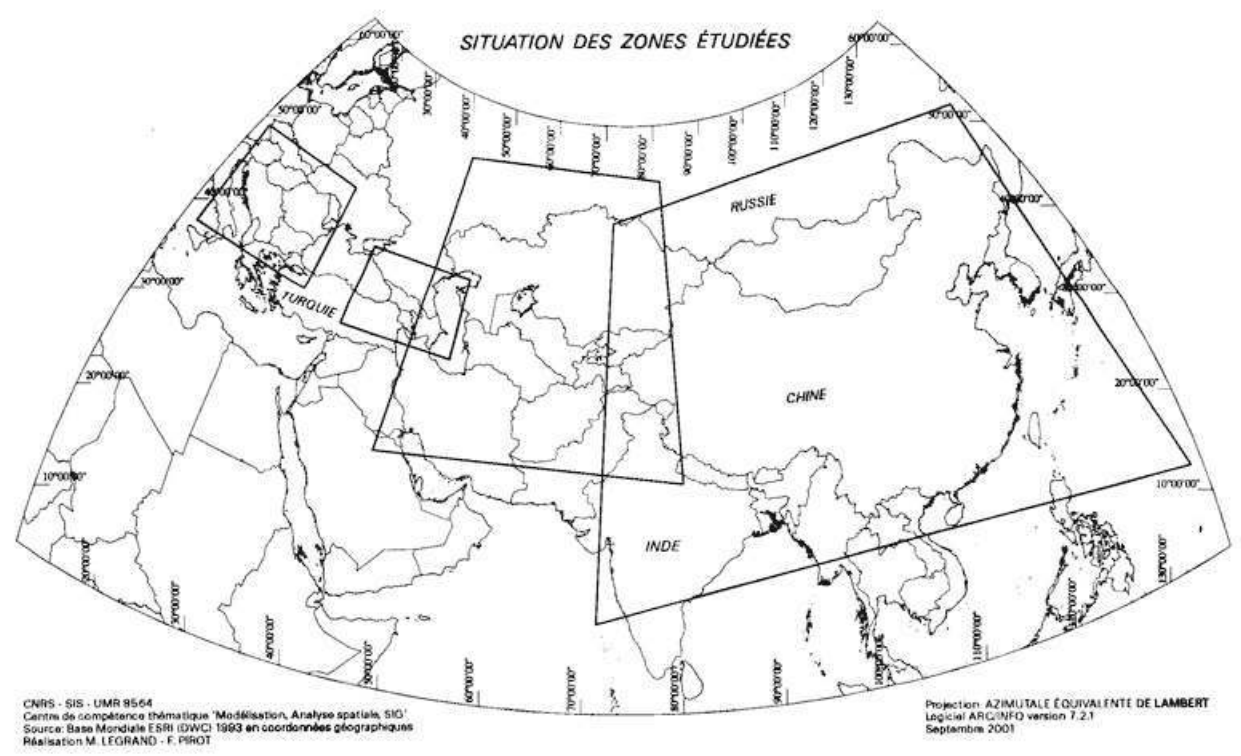

Projection AZIMUTALE ÉQUIVALENTE DE LAMBERT

Logiciel ARC/INFO version 7.2.1

Septembre 2001

CNRS - SIS - UMR 8564

Centre de compétence thématique « Modélisation, Analyse spatiale, SIG » Source : Base mondiale ESRI (DWC) 1993 en coordonnées géographiques

Réalisation : M. Legrand, F. Pirot

Pour dépayser d'emblée le lecteur et le remettre dans le contexte d'un régime communiste encore en vigueur, nous tournons d'abord notre regard vers la Chine avec l'article remarquablement informé d'Élisabeth Allès, Leïla Chérif-Chebbi et ConstanceHélène Halfon. Les minorités musulmanes s'y répartissent entre deux groupes numériquement comparables, mais vivant des situations contrastées. D'un côté les Hui, musulmans de langue et de culture chinoises, sont dispersés depuis plus d'un millénaire sur tout le pays ; bien qu'étant très minoritaires, ils sont bien intégrés; ne représentant pas aux yeux des autorités un danger pour l'unité du pays, ils sont relativement bien traités. De l'autre côté, les musulmans turcophones, qui sont principalement des Ouighours, et qui sont majoritaires dans la province du Xinjiang (Turkestan chinois) ; ils sont considérés par le gouvernement chinois comme un maillon faible de l'empire sur une frontière sensible ; la réaffirmation de leur identité ethnique ou religieuse est durement réprimée. L'article sur la Chine de ce recueil, bien que centré sur les Hui, dessine en filigrane la situation précaire des turcophones du Xinjiang; elle n'est pas sans rappeler celle que connurent naguère les turcophones du Turkestan (ou Asie centrale) russe à l'époque soviétique. Les Hui au contraire ont pu depuis 1980 reconstruire leurs institutions religieuses et culturelles, et réaffirmer de façon ostensible leur identité... ou 
plutôt leurs identités, puisque la nouvelle politique libérale a fait resurgir les clivages internes, et fait renaître les conflits avec les autres communautés religieuses du pays.

5 Les cinq républiques d'Asie centrale ex-soviétiques (Tadjikistan, Kirghizistan, Ouzbékistan, Turkménistan et Kazakhstan) sont ici magistralement évoquées par Olivier Roy. L'islam y était ressorti exsangue des campagnes systématiques d'éradication lancées à partir de 1927, en dépit d'une tardive reconnaissance en 1941, pour raisons de guerre, d'un islam officiel encadré par le mufti de Tachkent. La fin de la tutelle en 1991 donna le signal d'une spectaculaire réislamisation. Elle ne signifia pas pour autant la démission des nouveaux États : au contraire, leur contrôle sur les institutions religieuses s'est renforcé ; et seule une frange islamiste - notamment en Ouzbékistan et dans une moindre mesure au Tadjikistan - défie l'autorité des États en se liant à la nébuleuse afghano-pakistanaise et en combattant avec les Tâlebân.

L'Afghanistan, ici évoqué par ce fin connaisseur du pays qu'est Gilles Dorronsoro, représente un cas à part, mais significatif. Zone-tampon entre l'empire russe et l'empire anglais des Indes, il avait gardé son indépendance; mais la construction étatique y avait été embryonnaire. Le régime communiste introduit tardivement en 1978, et soutenu l'année suivante par l'armée soviétique, a produit un effet inverse de celui qui était souhaité : loin d'éradiquer ou d'encadrer les institutions musulmanes, il a provoqué une révolte dont les religieux ont pris le leadership et qui a abouti à la création d'un embryon d'État clérical administré par des étudiants en religion, les Tâlebân, formés dans les madrasa, ou séminaires, du Pakistan. Mal stabilisé, l'Afghanistan est devenu un abcès de fixation de l'islamisme terroriste mondial qui a été encouragé en sous-main par les services secrets pakistanais, et que l'Occident et l'Arabie Séoudite ont bien imprudemment encouragé et financé à l'origine pour lutter contre les Soviétiques.

7 Même après avoir abandonné l'Asie centrale, l'actuelle fédération de Russie abrite des minorités musulmanes qui ont leurs républiques autonomes; elles représentent, selon les estimations, de 8 à 20 millions d'âmes. Elles se regroupent autour de deux pôles: les musulmans de la Volga (avec leurs diasporas sibériennes) qui furent les premiers annexés par la Russie dès le milieu du XVI e siècle ; les musulmans du Caucase, conquis seulement au milieu du XIX ${ }^{e}$ siècle, lors des derniers empiètements de la Russie sur l'empire ottoman. Deux articles de ce numéro les concernent.

8 À l'Est de Moscou, la République des Tatars de la Volga centrée sur la ville de Kazan est le territoire musulman le plus anciennement annexé par la Russie en 1552. Il est célèbre aussi comme le plus ancien foyer du modernisme musulman, le Djadidisme, qui commença là dès la fin du XVIII ${ }^{e}$ siècle et se prolongea vers l'Asie centrale. La situation actuelle de l'islam dans cette république est présentée dans ce numéro par un Tatar, Räfyq Möhämmätshin qui appartient lui-même à l'appareil d'État: il fut conseiller du Mufti de Kazan et travaille aujourd'hui à l'Institut de l'Encyclopédie. Son approche est certes différente de celle des autres contributeurs qui ne sont pas partie prenante des phénomènes étudiés ; sa contribution n'en constitue pas moins un témoignage éclairant. Plusieurs constatations en ressortent. D'abord, à la différence de ce qui s'est passé en Asie centrale, l'islam officiel du Tatarstan a échoué à contrôler le renouveau islamique; l'interprétation de l'islam est liée au problème central de la Tatarie, qui est la revendication d'une souveraineté, laquelle peut se justifier de plusieurs façons. Pour la majorité des Tatars, cette revendication s'appuie sur un islam moderniste dans la lignée $\mathrm{du}$ Djadidisme. D'autres courants marginaux prennent deux directions fondamentalement opposées: soit le refus de l'islam à travers l'athéisme ou le néo- 
paganisme; soit au contraire le recours au fondamentalisme musulman qui prône le « combat sacré », le jihâd, pour l'établissement d'un État islamique.

9 Ce fondamentalisme, qui reste marginal au Tatarstan, passe au contraire au premier plan au Sud-Ouest de la Russie, dans le Caucase du Nord. Cette région a une tradition quasicontinue de résistance à l'impérialisme russe depuis son annexion il y a un siècle et demi, et la résistance acharnée de Maulana Shamil qui fut vaincu en 1859. Deux articles de ce recueil traitent de cette région. Le Caucase du Nord, qui comprend en particulier la Tchétchénie, est évoqué par Uwe Halbach. Ce dernier rappelle bien sûr l'héritage de la résistance caucasienne; mais il relativise les interprétations extrêmes entretenues de façon intéressée à la fois par la propagande de l'État russe et par les media occidentaux. En fait la montée en puissance de l'islamisme en Tchétchénie et dans les républiques voisines résulte plus de conflits ethniques et politiques mal gérés que d'une suprématie des radicaux: ces derniers restent en effet une minorité agissante appuyée sur des bastions situés dans les montagnes du Sud de la Tchétchénie.

Le Caucase du Sud est évoqué dans l'article de Raoul Motika qui traite de l'Azerbaïdjan. Alors que les autres régions étudiées dans ce recueil sont majoritairement sunnites, cette république ex-soviétique est pour environ deux tiers peuplée de turcs azéris chiites; ils sont historiquement très liés, du point de vue religieux et culturel, à l'Iran qui renferme aussi une minorité azérie. La domination russe, qui a duré un siècle et demi, a considérablement sécularisé les musulmans de cette république : l'islam a moins de place qu'en Iran dans leur affirmation identitaire et passe après le nationalisme turc et le modernisme. Deux autres différences sont tout aussi remarquables : l'importance prise par les femmes dans la vie sociale et même dans la transmission de la tradition islamique ; l'effort constant fait par les autorités religieuses pour la coopération entres chiites et sunnites. L'écroulement du système soviétique entraîna un renouveau religieux: les mosquées se sont multipliées, et l'enseignement religieux a été développé avec la création d'une Université islamique en 1992 ; le culte des tombes des saints a connu un regain qui n'est pas sans implications financières et politiques. Mais, malgré une intense propagande à partir de la République islamique d'Iran, relayée par un petit Parti islamique en Azerbaïdjan même, ce renouveau ne suivit pas les voies de l'islamisme chiite militant; de même la propagande des Wahhabites et des fondamentalistes sunnites de Turquie est restée marginale. À l'opposé, les intellectuels modernistes sont influents. L'Azerbaïdjan a en effet été déclaré un État laïque où toutes les religions sont en droit égales; mais les organisations religieuses restent étroitement surveillées grâce à un système d'encadrement hérité de l'Union Soviétique; et les missionnaires étrangers (islamistes comme évangélistes) ont été bannis en 1996. L'islam reste cependant un ingrédient indispensable dans la définition de l'identité azérie et dans la légitimation du pouvoir en place.

11 Les Balkans constituent le troisième pôle musulman à avoir connu la domination communiste : ils sont passés derrière le rideau de fer après la Seconde Guerre mondiale. L'histoire de cette région a été particulièrement tourmentée depuis 1945 : certains pays comme la Bulgarie restèrent dans l'orbite soviétique ; la Yougoslavie au contraire, avec Tito, choisit une voie séparée en 1948. L'Albanie, ballottée entre l'URSS et la Yougoslavie, et éphémèrement alliée à la Chine, amorça finalement en 1961 une évolution originale, marquée notamment par un radicalisme antireligieux extrême qui n'est pas sans rappeler les excès de la Révolution culturelle de la Chine. C'est aussi dans l'espace balkanique que la dé-communisation fut la plus conflictuelle, mettant au premier plan la confrontation 
entre musulmans et chrétiens catholiques ou orthodoxes dans l'ex-Yougoslavie (BosnieHerzégovine, Kosovo et Macédoine) et dans l'Albanie proprement dite.

La Bulgarie, objet ici d'une étude particulièrement fouillée de Nadège Ragaru, représente le cas d'une transition douce à l'ère post-communiste. Libérés des rigueurs d'une politique assimilationniste particulièrement rigoureuse, les musulmans bulgares jouissent d'une relative liberté : les tentatives de renforcement du pouvoir étatique ont fait long feu; les limites à la réislamisation viennent plutôt de la communauté musulmane handicapée par sa pauvreté et ses discordes. Dans cette réislamisation lente, le modèle traditionaliste de la vie islamique, marqué de soufisme, de syncrétisme et de modernisme, a plus de faveurs - notamment chez la majorité des musulmans d'origine turque - que le modèle fondamentaliste. Ce dernier peut séduire quelques Pomaks bulgarophones, lesquels sont également courtisés par des missionnaires orthodoxes qui veulent les reconvertir. Les Tsiganes musulmans, particulièrement frappés par la crise économique, sont encore plus vulnérables à la rechristianisation qui leur est proposée cette fois par des pasteurs évangélistes.

En ce qui concerne l'espace albanais, Nathalie Clayer démêle de façon nuancée une situation complexe. L'islam, dévalorisé sous les régimes communistes, n'est pas le moteur premier des conflits. Néanmoins deux développements récents ont renforcé le poids de l'islam dans l'affirmation nationaliste albanaise : premièrement la montée du conflit avec les Serbes au Kosovo et avec les Macédoniens en Macédoine; et deuxièmement le déplacement de l'affirmation nationaliste albanaise de chez les Tosks de l'Albanie du Sud vers les Gegs du Nord de l'Albanie et du Kosovo.

Enfin, Xavier Bougarel, auteur d'une thèse sur l'islam et la politique en BosnieHerzégovine, clarifie le débat - obscurci par trois années de guerre - sur les rapports de l'islam à l'identité nationale et à la politique dans cette région meurtrie de l'ancienne Yougoslavie. À travers une étude rigoureuse de trois penseurs aux orientations contrastées, il présente les trois principales interprétations de l'islam qui ont cours dans le nouvel État; elles présentent l'islam soit comme une foi individuelle (Fikret Karčić), soit comme une culture commune (Enes Karić), soit enfin comme une idéologie politique discriminante (Adnan Jahić). La dernière interprétation, nettement fondamentaliste, a bien pu avoir la préférence officielle à la faveur de la guerre; elle est cependant loin d'être la seule, ni même de rallier la majorité de la population musulmane.

Pour discerner les grandes tendances qui se dégagent de ces évolutions du monde communiste ou ex-communiste, nous poserons quatre questions. Quelle est l'extension et la profondeur du renouveau islamique? Dans quelle mesure est-il freiné ou encadré par les autorités étatiques? Quelles interprétations de l'islam tendent à s'imposer dans les idéologies et dans les institutions? Le renouveau favorise-t-il la concorde entre musulmans, et entre musulmans et non-musulmans? Ou bien ouvre-t-il la voie aux conflits identitaires?

16 Le renouveau islamique apparaît comme un fait massif dans tous les cas étudiés dès les années 1990-1991 qui virent la dissolution du communisme soviétique et balkanique, souvent même, comme en Chine, dès la décennie d'ouverture qui précéda. L'analyse de ce renouveau est plus ou moins poussée selon les contributeurs: l'inventaire particulièrement soigné de l'article sur la Chine peut servir de guide. Les deux grands traits de ce renouveau sont la reconstruction interne, et l'ouverture sur le monde extérieur. À l'intérieur le trait le plus spectaculaire est la restauration des mosquées dont la plupart avaient été fermées, et même la construction de nouvelles mosquées : en Chine, 
en Asie centrale, en Russie, dans les Balkans, partout le nombre de mosquées ouvertes a fait plus que décupler. Même si les statistiques tendent à être gonflées par les intéressés, la prolifération des édifices de culte est une tendance avérée. Cette réouverture des lieux de culte pose le problème du personnel religieux qu'il a fallu improviser en faisant sortir de l'ombre le clergé "parallèle» qui s'était maintenu pendant la persécution, en improvisant de nouveaux mollahs, ou en les important de l'étranger; il a surtout fallu reconstruire les institutions d'enseignement chargées de former ces nouveaux cadres religieux. En Chine on assiste même à la naissance d'un enseignement général confessionnel destiné à se substituer à l'enseignement d'État en crise. Tout cela va avec l'expansion de la littérature musulmane à nouveau autorisée: manuels d'instruction religieuse, presse confessionnelle, ou même littérature générale comme en Chine.

Cette reconstruction interne est inséparable d'une ouverture extérieure: les contacts avec les autres pays musulmans étaient à l'époque communiste sévèrement réglementés et instrumentalisés pour la propagande d'État vers les pays du Moyen-Orient afin de se donner une façade libérale masquant les persécutions intérieures. Les restrictions ont sauté au cours des années 1980-1990, ce qui a eu une double conséquence. D'un côté, l'espace intérieur s'est brusquement ouvert: une multitude d'associations missionnaires musulmanes comme la Tablîghî Jamâ'at, d'ONG islamiques, et de fondations plus ou moins liées au Moyen-Orient se mirent à quadriller les pays communistes et ex-communistes récemment ouverts; on trouvera une liste quasi-exhaustive de ces organisations musulmanes venues de l'extérieur dans l'article de Nathalie Clayer sur l'espace albanais. Elles sont d'ailleurs souvent en concurrence avec des missionnaires protestants. Les gouvernements des pays d'origine sont souvent présents derrière ces réseaux, et au premier chef l'Arabie Séoudite qui distribue sa manne de pétro-dollars pour favoriser l'interprétation wahhabite de l'islam que l'on peut, à juste titre, qualifier de "pétroislam ». L'Iran, pourtant géographiquement proche de l'Asie centrale et de la Russie, a eu un impact relativement limité (sauf peut-être en Bosnie et en Albanie) à cause de la barrière sectaire qui sépare les Iraniens chiites de populations majoritairement marquées par le sunnisme hanafite turc; même dans l'Azerbaïdjan, qui est limitrophe de l'Iran et aux deux tiers chiite, la propagande de la République Islamique a eu peu de succès, car elle heurte la sensibilité d'une population sécularisée en profondeur par un siècle et demi de domination russe. En sens inverse, les voyages depuis les pays étudiés ici vers les pays musulmans se sont multipliés pour le pèlerinage à la Mecque et pour les études, débordant très largement les contingents fixés par les gouvernements qui sont impuissants à encadrer l'intensification de ces échanges.

Car - et c'est notre deuxième point - la libéralisation n'a pas empêché les États de chercher à contrôler la vie religieuse. Comme le fait justement remarquer Olivier Roy à propos de l'Asie centrale, les nouveaux gouvernements ont repris, nationalisé et développé les modèles soviétiques de contrôle de la vie religieuse à travers une hiérarchie cléricale officielle. Le succès fut-il à la mesure des ambitions ? C'est dans l'Asie centrale qui a le mieux conservé les personnels et les structures de l'Union soviétique - que le succès fut le plus franc, conclut Olivier Roy, car les États y ont clairement gagné le contrôle de l'islam officiel, sans se laisser déborder par les circuits parallèles. En Bosnie, comme le montre Xavier Bougarel, des structures de contrôle analogues à celle de l'ancien modèle soviétique, avec la prééminence du parti islamiste SDA, sont dénoncées par l'opposition. Ailleurs les succès de l'État sont beaucoup plus mitigés. Ainsi l'Association Islamique de Chine, qui incarne le contrôle gouvernemental, est débordée 
par les initiatives privées; elle échoue à canaliser les initiatives individuelles et la multiplication des échanges avec l'étranger. Une évolution similaire se constate dans deux autres régions de l'ancienne zone d'influence soviétique: Räfyq Möhämmätshin montre comment deux structures officielles rivales se neutralisent au Tatarstan; Nadège Ragaru explique aussi comment en Bulgarie un encadrement officiel qui se voulait contraignant est délégitimé et rendu inefficace par les compétitions internes pour le leadership de la communauté musulmane. Il est deux cas enfin où l'encadrement officiel a franchement échoué. L'Afghanistan d'abord : isolé du monde moderne, il a des structures étatiques inchoatives; comme le montre la première partie de l'article de Gilles Dorronsoro, les essais de l'encadrement de l'islam à l'époque de l'occupation soviétique, qui prolongeaient la politique de l'ancienne monarchie, aboutirent à une révolte généralisée au nom de l'islam. En Tchétchénie, selon Uwe Halbach, l'alliance de la Russie avec les musulmans modérés du Nord n'a pas pu faire contrepoids aux irrédentistes des montagnes dont la rébellion a fait de nouveau tache d'huile et entrainé une seconde guerre.

19 La montée du radicalisme dans ces régions marginales que sont l'Afghanistan, la Tchéchénie et la Bosnie nous amène à notre troisième question : dans quel sens s'effectue le renouveau islamique dans les pays communistes et ex-communistes? On ne peut, en lisant ce recueil, manquer de remarquer le caractère voyant des publications islamistes, comme les œuvres de Maududi et de Qutb, qui sont abondamment diffusées de la Chine aux Balkans. Les partisans d'un islam pur et dur, amateurs de jihâd, font beaucoup parler d'eux et ont souvent été financés et encouragés, en Afghanistan et en Bosnie, par l'Arabie Séoudite et même par les pays occidentaux. L'alliance contre nature de l'islamisme avec les pétro-dollars saoudiens a donné lieu à la popularisation en Russie et en Asie centrale du vocable « wahhabite » qui désigne tous les musulmans radicaux. Cette grande visibilité des islamistes signifie-t-elle pour autant qu'ils ont dominé le mouvement de réislamisation de l'aire post-communiste? Ce n'est pas si sûr. Nous sommes en fait en présence de minorités agissantes qui ont l'art de se faire entendre et de se faire remarquer, faisant tomber les media occidentaux, voire les chercheurs, dans leur piège. Le paysage social et religieux, comme le montre en particulier l'article de Xavier Bougarel, est beaucoup plus complexe et modéré. L'aire considérée ici n'a jamais été réellement mise en danger par l'islamisme. Ainsi l'étude fouillée d'olivier Roy montre bien qu'il reste marginal en Asie centrale : là où il est présent, il est soit résorbé dans le processus de construction nationale comme au Tadjikistan, soit poussé dans la clandestinité et chassé vers l'Afghanistan comme dans le cas de l'Ouzbékistan. On est loin ici des fantasmes médiatiques, parfois encouragés par la propagande des pays concernés, selon laquelle l'aire étudiée ici aurait été menacée par une déferlante islamiste. Au contraire l'islamisme y est resté marginal, et ce qu'il faut plutôt expliquer, ce sont les cas où il a marqué des points: l'Afghanistan, la Tchétchénie et la Bosnie. Pour les deux premiers pays, deux raisons de ces succès apparaissent. Il s'agit d'abord de régions marginales par rapport à l'empire russe. En second lieu, elles n'ont connu que des structures étatiques très lâches: elles n'ont jamais été intégrées dans des empires modernes car l'Afghanistan est resté jusqu'en 1978 une sorte de no man's land, et la Tchétchénie a été en rébellion quasi-permanente depuis sa conquête. La Bosnie représente un autre cas de figure : la poussée islamiste, qui y fut plus limitée entre 1992 et 1995, a été rendue possible par un effondrement temporaire de l'Etat. Ailleurs, et même finalement en Bosnie et en Tchétchénie, l'islamisme - si visible qu'il soit - est loin de représenter la forme d'islam dominante. Les majorités silencieuses incarnent un islam 
plus traditionnel et plus œcuménique, teinté de soufisme et accueillant aux coutumes locales. Enfin une large frange éduquée, celle de «l'islam citoyen " pour reprendre encore une expression en vogue dans les Balkans, prône une forme plus moderniste et plus individualiste de la religion.

Si danger il y a - et là nous abordons notre quatrième et dernière question - il ne réside pas dans l'islamisme, mais dans les nouvelles formes d'affirmation des identités ethniques et religieuses qui se sont manifestées à l'occasion de la libéralisation et de l'effondrement des régimes communistes. N'ayant plus à faire front contre un ennemi idéologique, les communautés religieuses et ethniques se dressent plus facilement les unes contre les autres en dessinant des fractures internes. Le phénomène est visible, sans drame jusqu'ici, en Chine où renaissent les affrontements d'antan entre confréries soufies, entre traditionalistes et fondamentalistes; il y a d'autre part recrudescence de la confrontation entre musulmans et non musulmans. En Asie centrale et en Afghanistan comme au Caucase, les conflits s'entrecroisent sans que l'on sache s'il faut donner la priorité aux interprétations idéologiques ou ethniques; le facteur ethnique semble cependant incontournable entre Tadjikistan et Ouzbékistan. On note, d'autre part, en Bulgarie un durcissement des stéréotypes ethniques, notamment à l'encontre des Tsiganes. Mais c'est sur le territoire de l'ex-Yougoslavie et de l'Albanie que le durcissement des frontières dites « ethniques » a fait le plus de ravages : l'établissement d'une démarcation en Bosnie entre les catholiques, les orthodoxes et les musulmans - qui ont participé récemment d'une culture commune - pour en faire des nationalités distinctes (Croates, Serbes et Bosniaques/Bochniaques) a conduit aux événements tragiques que l'on sait. Les troubles au Kosovo puis en Macédoine et en Albanie, avec le durcissement d'une identité albanaise parfois centrée sur l'islam (bien que tous les Albanais ne soient pas musulmans), n'a fait qu'approfondir le fossé entre chrétiens et musulmans. De même, à l'intérieur de la communauté musulmane, des fossés se creusent, notamment la résurgence en Albanie du clivage entre Gegs et Tosks.

21 Si le péril islamiste doit être ramené à ses justes proportions, les problèmes posés par les dérives identitaires, à la fois religieuses et ethniques, sont bien plus larges. D'autre part, ils relèvent plus d'une histoire sur le long terme que d'une analyse politologique ponctuelle. Ils doivent alors être traités dans le cadre d'une vision comparatiste plus large que celle qui se limite au monde (ex-)communiste. L'étude amorcée ici gagnerait à être étendue aux phénomènes identitaires qui ont marqué la dissolution des empires coloniaux en Inde, en Indonésie ou en Afrique où l'on retrouve, souvent inextricablement mêlés, les phénomènes religieux et ethniques.

\section{NOTES}

1. Le présent recueil et l'ouvrage intitulé L'Islam oriental du Caucase à la Chine (coordonné par Andrée Feillard, Paris, La Documentation française, à paraître en 2001) sont les deux derniers ouvrages collectifs préparés dans le cadre du Groupe de recherche sur le monde musulman périphérique (PRI de l'EHESS et GDR $\mathrm{n}^{\circ} 122 \mathrm{du}$ CNRS). Ce groupe, qui a fonctionné dans le cadre 
de l'EHESS de 1982 à 2001, est maintenant prolongé par le programme « Minorités en miroir » de l'Institut d'Études de l'Islam et des Sociétés du Monde Musulman (IISMM) de l'EHESS. Les Lettres d'information publiées par le Groupe, vont être rééditées en un volume, aux Éditions Isis d'Istanbul, en 2002 (par les soins de Stéphane A. Dudoignon et Catherine Servan-Schreiber). Le présent numéro a été bouclé avant l'attentat du 11 septembre 2001 contre le World Trade Center de New York : les contributeurs n'ont pas pu prendre en considération les conséquences de cet attentat sur les régions étudiées et les réinterprétations qu'elles entraînent.

\section{AUTEURS}

\section{MARC GABORIEAU}

Centre d'Études de l'Inde et de l'Asie du Sud, EHESS-CNRS

\section{ALEXANDRE POPOVIC}

Centre d'Études du domaine turc, EHESS-CNRS 